\title{
Perspectivas y trayectorias teóricas \\ de la Antropología de la Infancia \\ Finales del siglo XIX y siglo XX
}

\section{Diana Marcela Aristizábal García*}

dm.aristizabal65@uniandes.edu.co

\begin{abstract}
Resumen
Este artículo hace un recorrido teórico por las principales contribuciones que las Ciencias Sociales y la Antropología han hecho a los estudios sociales sobre la infancia y específicamente al campo de la "Antropología de la Infancia". Se argumenta que el cambio de mirada antropológica hacia la infancia es el producto de una trayectoria académica que no se puede comprender aisladamente desde la misma disciplina. Por el contrario, este campo de estudio está estrechamente conectado con los debates sobre la infancia que se han desarrollado desde la filosofía, la biología, la psicología, la sociología, la historia y los estudios feministas, con los cuales se han trazado unas rutas teóricas compartidas, a la vez, que cada disciplina ha diseñado sus propias apuestas para la comprensión y la teorización sobre la infancia.
\end{abstract}

\section{Palabras claves}

Antropología de la infancia; estudios sociales de la infancia; siglo XIX, siglo XX

\section{Perspectives and theoretical trajectories of the Anthropology of Children Late nineteenth and twentieth century}

\begin{abstract}
This article makes a theoretical journey through the main contributions that Social Sciences and Anthropology have made to social studies about childhood and specifically to the field of Anthropology of Childhood." This essay argues that the change of anthropological view towards childhood is the product of an academic trajectory that cannot be understood in isolation from the same discipline. On the contrary, this field of study is closely connected with the debates on childhood that have developed from disciplines such as biology, psychology, sociology, history and feminist studies, with which shared theoretical paths have been traced, at the same time, that each discipline has designed its own bets for understanding and theorizing about childhood.
\end{abstract}

\section{Keywords}

Anthoropology of Childood; Social studies about childhood; XIX century, XX century 


\section{Introducción}

La relación entre antropología e infancia no es nueva. Desde sus inicios como disciplina moderna, la antropología y varios de sus pioneros como Bronislaw Malinowski, Franz Boas, Margaret Mead y Ruth Benedict registraron en sus relatos etnográficos la presencia de niños tanto en comunidades occidentales, como no occidentales. Así, por ejemplo, Malinowski, representante de la tradición antropológica funcionalista británica, describió "En la vida sexual de los salvajes en el noroeste de Melanesia" (1929) las ideas y prácticas de los Trobriand en relación a la sexualidad y cuestionó la generalización de la noción freudiana del complejo de Edipo. En sus notas de campo relató cómo en la crianza de los niños participaban tanto los padres, como las madres y precisó varios elementos sobre el comportamiento de los niños: eran libres e independientes, tenían varios juegos sexuales que no tenían "ninguna interferencia de personas mayores" (MALINOWSKI, 1929, pág. 50) y aunque a veces los padres golpeaban a sus hijos, también "muy a menudo ellos golpeaban a sus padres" (MALINOWSKI, 1929, pág. 45).

Por su parte, Franz Boas considerado el fundador de las escuelas norteamericanas del Particularismo Histórico y de Cultura y Personalidad registró en "La mente del hombre primitivo" (1911) las diferencias físicas y de crecimiento entre grupos de niños pertenecientes a diferentes etnias y grupos sociales. En una de sus investigaciones planteó que "los niños de todas las razas ofrecen indudablemente analogías de desarrollo dependientes del desarrollo del cuerpo y diferencias concordantes con las demandas exigidas por su gradual iniciación en la cultura en que viven" (BOAS, 1911, pág. 182). Si bien la observación de los niños y de sus prácticas cotidianas no fue el centro de observación y de análisis antropológico, ni tampoco los niños se con- sideraron los principales informantes, varios antropólogos pioneros como Boas y Malinowski describieron informes valiosos, aunque fragmentarios, sobre la vida de los niños en diferentes contextos culturales. A la vez, esta literatura etnográfica temprana proporcionó evidencia inicial de las amplias variaciones culturales de los grupos humanos a través de la observación de los niños, las prácticas de crianza y las relaciones entre niños y adultos (LEVINE, 2007, pág. 249).

Uno de los debates recientes sobre el tema lo han planteado los antropólogos Lawrence Hirshfeld (2002), Heather Montgomery (2009) y David Lancy $(2012,2015)$ en relación a la tradición de estudio antropológico sobre la infancia. Hirshfeld argumentó que pocos trabajos importantes en antropología se han centrado específicamente en los niños y esta disciplina "ha mostrado poco interés en ellos y en sus vidas" (HIRSHFELD, 2002, pág. 612). Señaló que esto es producto de una visión empobrecida del aprendizaje cultural que sobrevalora el papel de los adultos y subestima la contribución de los niños a la reproducción cultural (HIRSHFELD, 2002, pág. 612).

Frente a esta tesis, Montgomery y Lancy han mostrado un acumulado de estudios antropológicos, que desde diferentes perspectivas (social, biológica, arqueológica), directa o indirectamente, han estudiado ciertos aspectos de la vida de los niños. Ambos autores han señalado que los avances en este campo han estado dispersos en trabajos sobre familia, parentesco, prácticas de crianza, socialización, enculturación y desarrollo (LANCY, 2012, pág. 3), que fueron los temas centrales de investigación antropológica desde finales del siglo XIX hasta las primeras décadas del siglo XX. Así, no solo la antropología, sino el conjunto de las Ciencias Sociales, entendidas como productos históricos, respondieron durante esta época a unas agendas acadé- 
micas y a unos intereses de investigación en las cuales los niños no podían ser pensados como el centro de investigación. Esto no implica necesariamente que pueda afirmarse que la antropología y las ciencias sociales “abandonaron", “olvidaron" y "marginalizaron" voluntariamente a los niños, como lo sugirió Hirshfeld.

En este sentido, la versión "de la antropología centrada en el niño" o el campo de la "Antropología de la Infancia”, como se le conoce desde finales de la década del ochenta del siglo XX, es producto de un acumulado de conocimiento previo y de unos debates académicos y políticos que hicieron posible que la infancia se desplazara desde los márgenes de la literatura antropológica y se situara en el centro de los proyectos de investigación. Tal como lo plantea la antropóloga Heather Montgomery, este cambio de mirada "no significó centrarse solo en los niños, ni construir una subcultura de la sociedad donde los niños existen aparte de las familias y las comunidades. Más bien significó que la investigación sobre los niños intentaría comprender sus perspectivas y sus vínculos con sus familias" (MONTGOMERY, 2000, pág.16).

En este sentido, este artículo discutirá cómo la antropología de la infancia se ha constituido como un campo de estudio que se ha nutrido de una trayectoria teórica y epistemológica de varios saberes y disciplinas, a la vez, que ha propuesto unas trayectorias propias para comprender la infancia. Es imposible pensar el desarrollo de este campo sin tener en cuenta los debates políticos que se han presentado desde las primeras décadas del siglo XX sobre el lugar social que tienen los niños, los cuales se materializaron en un conjunto de políticas como la Declaración de Ginebra de los Derechos del Niño (1924), la Declaración de los
Derechos del Niño de las Naciones Unidas (1959) y la Convención sobre los Derechos del Niño (1989). Así, se analizarán los efectos que ha tenido para el estudio antropológico de los niños las definiciones sobre la infancia presentadas por la legislación internacional, se plantearán cuáles han sido los principales aportes de la antropología a los estudios sociales sobre infancia y cuáles son actualmente las propuestas para estudiar la vida de los niños. Por último, se mostrarán algunas de las líneas de investigación más importantes que se han consolidado en el contexto de América Latina y los aportes de la región a la discusión.

\section{Las primeras construcciones discursivas sobre la infancia}

La Antropología, como las demás Ciencias Sociales, no iniciaron sus reflexiones sobre los niños en el vacío. Desde el siglo XVII se han producido una serie de discursos occidentales sobre lo que es un niño, los cuales han permeado la comprensión de la noción de infancia, no solo en la academia, sino también en las políticas públicas. Algunas de las construcciones teóricas más importantes fueron las propuestas por los filósofos europeos Thomas Hobbes, John Locke y Jacques Rousseau ${ }^{1}$. El filósofo inglés Thomas Hobbes en su famoso tratado político "El Leviatán" (1651) propuso pensar que los seres humanos, incluyendo los niños, son esencialmente malvados. Los niños nacían en un estado anárquico y sin reglas, por lo que era responsabilidad de los padres controlar los instintos, a través de la disciplina, las reglas y el control estricto.

En contraposición a esta forma de comprender la humanidad, el filósofo inglés Jhon Locke en sus

\footnotetext{
${ }^{1}$ Algunos autores que han desarrollado una discusión más a profundidad sobre estos debates son Montgomery (2003), Hogan (2005), Kehily (2009) y Messenger (2010).
} 
libros "Ensayo sobre el entendimiento humano" (1690) y "La conducta del entendimiento" (1693) rechazó cualquier alusión al postulado sobre las "ideas innatas" de la bondad y la maldad que tendrían los niños al nacer. A cambio, planteó que el niño nacía como una “tabula rasa” y que con instrucción y educación, podría convertirse en un ser humano completo y racional, es decir, un adulto (Montgomery; Woodhead, 2003, págs. $63-64)$.

A estas tesis, se les sumó un siglo después el postulado del filósofo francés Jacques Rousseau que en "Emilio o de la Educación" (1762) planteó que los niños eran naturalmente inocentes, puros y buenos y que era a través de la experiencia en el mundo que los humanos aprendían la maldad y eran corrompidos. En uno de los apartados de su texto afirmaba: "todo se degenera en manos del hombre" (ROUSSEAU, 1762, pág. 37, edición de 1998). Rousseau fue uno de los primeros en plantear que la infancia tenía varias etapas que se desarrollaban desde el momento del nacimiento hasta los 12 años de edad (MESSENGER, 2010, pág. 26).

Estos tres discursos filosóficos sustentan todavía muchas de las discusiones académicas y políticas contemporáneas sobre la infancia, en términos del carácter de los niños, sus posibilidades de acción, limitaciones, características físicas, emocionales e intelectuales. Aunque estos primeros discursos occidentales ya planteaban una diferenciación de la infancia como una etapa particular y de naturaleza diferente a la adultez, fue hasta finales del siglo XIX y durante las primeras décadas del siglo XX que la infancia pasó a ser un “objeto" de interés para el Estado, la economía, el mercado, las políticas públicas y para disciplinas modernas como la psicología, la pedagogía, la medicina y la antropología (PEDRAZA, 2008). En parte esto se dio por varias circunstancias históricas y sociales que suscitaron el interés público por la infancia: las campañas para luchar contra la mortalidad infantil, (producto de las enfermedades y la desnutrición); los movimientos de abolición del trabajo infantil; la creación de auspicios, escuelas y hogares para el cuidado y la enseñanza de los niños y, como consolidación política de estas preocupaciones, la Declaración de los Derechos de los Niños en 1924.

A finales del siglo XIX, la antropología como naciente disciplina, estuvo fuertemente influenciada por varios saberes, especialmente la biología y la psicología, que se convirtieron en la base para sus posteriores desarrollos teóricos sobre la infancia. La teoría evolucionista de Charles Darwin (1859) y sus estudios científicos sobre la infancia impulsaron el interés de otros científicos y médicos en estudiar el desarrollo físico, intelectual y moral de los niños. En su libro “Bosquejo Biográfico de un Niño" (1877), Darwin reflexionó sobre sus postulados evolucionistas en relación a la observación sistemática de sus propios hijos y planteó varias ideas sobre los patrones de crecimiento físico, en términos de una serie de categorías en conexión con la edad: acciones reflejo, visión, movimiento, afección, razón y sentido moral. Un ejemplo de sus observaciones es la siguiente: “es sorprendente cómo lentamente él adquiere el poder de seguir con sus ojos un objeto que se mueve, esto no lo podía hacer bien a los siete meses y medio de nacido" (DARWIN, 1877, pág. 118, edición de 1965).

El enfoque darwinista también sugirió que podrían hacerse comparaciones entre el desarrollo de la especie humana con el desarrollo de los niños hasta llegar a la adultez, idea que tuvo un gran impacto en ciertas teorías antropológicas, pues los niños comenzaron a ser científicamente interesantes, especialmente porque su desarrollo fue visto como una posibilidad de comprender la evolución de la especie humana y de su cultura. A partir de estos postulados, también varios 
psicólogos como Wilhelm Preyer, Stanley Hall y Arnold Gesell recurrieron a la observación sistemática de los niños, como forma de comprender la mente infantil, los comportamientos y patrones de conducta (Montgomery; Woodhead, 2003, pág. 98).

Así, desde finales del siglo XIX, la psicología basada en los postulados evolucionistas de Charles Darwin se convirtió en la primera disciplina que iniciaría un proyecto de estudio centrado en la infancia. El interés por el estudio sistemático del desarrollo infantil se consolidó en un contexto de urbanización e industrialización en el que reformadores políticos occidentales necesitaban evidencias científicas que soportaran el establecimiento de legislación sobre las edades para el trabajo infantil, los grados de escolarización, el matrimonio, la responsabilidad criminal y el derecho al voto (KEHILY, 2009, pág. 48). Varios expertos - maestros, pediatras, psicólogos - "estaban ansiosos por herramientas que pudieran aplicarse en la crianza, la formación y la enseñanza de las escuelas" (WOODHEAD, 2009, pág. 23).

Dos han sido fundamentalmente las líneas de investigación que siguió esta disciplina: el psicoanálisis y la psicología del desarrollo. Sigmund Freud, desde el psicoanálisis, planteó varias ideas sobre la mente infantil, el desarrollo psicológico, el juego y la sexualidad de los niños. Al igual que Darwin, Freud postuló que el desarrollo psicológico y sexual infantil estaba compuesto por varias fases (oral, anal, fálica, latencia genital), que todos los niños tenían en común durante los primeros seis años de vida. El trabajo de Freud se dirigió principalmente a "comprender la mente adulta mediante el rastreo de las experiencias de los niños" (HOGAN, 2005, pág. 32). Desde la segunda década del siglo XX las ideas de este autor se extendieron en el discurso público y plantearon preguntas sobre cuál era la mejor manera de criar un niño, qué era un niño normal y a través de qué etapas del desarrollo los niños llegaban a ser adultos. Muchas de sus teorías, se distorsionaron y se generalizó su aplicación a todos los contextos (LEVINE, 2007, pág. 248), cuestión que sería cuestionada por varios antropólogos, especialmente de la Escuela Cultura y Personalidad.

Por su parte, la psicología del desarrollo, una tradición académica diversa que es reconocida por los trabajos de psicólogos como Jean Piaget, Lev Vygotsky y Erik Erikson propuso diferentes interpretaciones sobre las etapas de desarrollo normal y típico que debían presentar los niños, no solo físicas, sino también motoras e intelectuales (WALKERDINE, 2009, pág. 58). Jean Piaget a través de la implementación de test y pruebas experimentales se concentró en indagar sobre el desarrollo intelectual de los niños y planteó que las etapas de aprendizaje eran un proceso natural y universal en todos los niños. Aunque Piaget consideraba que el desarrollo cognitivo era moldeado tanto por las influencias biológicas, como sociales y a través de métodos como las entrevistas intentó comprender aspectos de la vida cotidiana de los niños, su énfasis fue sobre todo cognitivo. En contraste, Vygotsky ubicó el desarrollo en términos más contextuales e históricos. Desde esta perspectiva más construccionista, Vygotsky propuso pensar el desarrollo infantil como un "producto de unos contextos económicos, sociales y culturales específicos” (MONTGOMERY, 2003, pág. 113).

Así, aunque no es posible pensar la psicología del desarrollo como una tradición unificada, en general, esta perspectiva produjo una versión de la infancia en la que predomina el interés por la progresión del niño hacia la vida adulta, a través del análisis de parámetros como la edad, el desarrollo físico y la adquisición de ciertas habilidades y competencias cognitivas según etapas. El niño, en este sentido, es comprendido en términos de transiciones: de la inmadurez a la madurez, 
de la irracionalidad a la racionalidad y de la incompetencia infantil a la competencia adulta. La práctica de monitorear la salud y el crecimiento de los niños y de pensar la infancia en términos de edades y etapas, de normalidad y anormalidad no solo se restringió a los profesionales de la salud física y mental, sino que desde las primeras décadas del siglo $\mathrm{XX}$ se convirtió en un paradigma fundamental en el ámbito educativo, para las políticas públicas e incluso se trasladó a los discursos cotidianos y domésticos, "pues muchos padres en contextos occidentales empezaron a compartir este nuevo conocimiento a través de manuales de crianza" (HARDYMENT, 1984).

De esta manera, durante las primeras décadas del siglo XX, la psicología del desarrollo infantil y el psicoanálisis se convirtieron en el paradigma occidental más dominante e influyente para las teorías sobre la infancia que empezaban a desplegarse en las ciencias sociales, especialmente provenientes de la sociología y la antropología. Algunos sociólogos trasladaron las nociones de la psicología del desarrollo a la teoría de la socialización o enculturación a través de la cual se pretendía comprender el modo en que los niños eran socializados a través instituciones sociales como la familia, el grupo de pares y la escuela y cómo funcionaba la transmisión de la cultura de una generación a otra. Desde esta perspectiva la sociedad moldea al individuo y aunque no se ignora el carácter biológico de los seres humanos, se ofrece una explicación especialmente social. En este sentido, el modelo de desarrollo, ya no está ligado a lo que el niño naturalmente y biológicamente es, como lo presentaría la teoría evolucionista, sino a lo "que la sociedad demande del niño y cómo este aprende a ajustarse a las normas sociales" (JENKS, 2009, pág. 49).

En una línea similar, varios antropólogos empezaron a interesarse por explorar los orígenes de los patrones culturales en la infancia y las prácticas de crianza. En parte esto explica por qué los primeros registros etnográficos del siglo $\mathrm{XX}$ en las que se mencionaron a los niños se enfocaron en temas relacionados con los procesos de socialización, como el parentesco, la crianza, el juego y las relaciones entre adultos y niños. Sin embargo, habría también que precisar que varios trabajos antropológicos pioneros como los de Franz Boas, Margaret Mead y Ruth Benedict de la Escuela Cultura y Personalidad si bien se apropiaron de varios elementos teóricos y metodológicos importantes planteados por la psicología del desarrollo, el psicoanálisis y la teoría de la socialización, también presentaron discusiones importantes que pusieron en cuestión una idea que estaba en el fondo de todas estas construcciones discursivas sobre la infancia desde Hobbes, hasta el paradigma de la socialización: una imagen occidental, singular y universal de la infancia y por tanto, de su desarrollo, sus características, sus formas de aprendizaje y sus relaciones con los adultos.

\section{Reflexiones antropológicas sobre los niños: finales siglo XIX - primeras déca- das siglo $\mathrm{XX}$}

La antropología como disciplina moderna no ha tenido un único camino para comprender la infancia. Desde finales del siglo XIX y durante las primeras décadas del siglo $\mathrm{XX}$, las dos grandes tradiciones antropológicas (británica y norteamericana) siguieron rutas diferentes para el estudio de los niños. Aunque la mayoría de estos primeros trabajos no hicieron alusión directa al estudio de la infancia, como ya lo hacía para el momento la psicología, ni tampoco se acudió a los niños como informantes centrales, esto no implica que los niños hayan sido ignorados: "los niños fueron usados por los antropólogos en una variedad de formas pa- 
ra entender mejor la sociedad en general. Los antropólogos usaron explícitamente ideas para pensar y discutir otros temas como las relaciones entre los primitivos, el pensamiento infantil y la evolución de la sociedad humana” (MONTGOMERY, 2009, pág.13).

Así, la tradición británica con antropólogos como Edward Tylor (1871), Jhon Lubbock (1878) y Dudley Kidd (1906) comenzó a introducir a los niños en sus relatos etnográficos con el fin de examinar la naturaleza de la evolución humana, sus habilidades lingüísticas y técnicas. Así, el niño se tomó como la imagen más cercana para estudiar la vida primitiva y salvaje y comprender el desarrollo cultural, en oposición al civilizado mundo europeo y norteamericano. Desde una perspectiva evolutiva y desarrollista, los niños se convirtieron para estos antropólogos en los sujetos más cercanos para comprender las etapas de la evolución de la especie humana. Eran los "otros" más fácilmente observables, "eran salvajes en casa" (MONTGOMERY, 2009, pág. 20).

Así, por ejemplo, Tylor afirmaba que los juegos de los niños podrían asimilarse con la forma en que los ancestros vivían: "los niños eran representativos de una cultura remota antigua" (TYLOR, 1871, pág. 73). En una línea similar, el antropólogo Dudley Kidd, al estudiar en 1906 aspectos de la vida cotidiana de los niños Kafir en el sur de África, planteó que "la infancia es el más importante instructivo e interesante periodo de la vida de un salvaje" (KIDD, 1906, pág. viii). Aun cuando hoy estas ideas puedan resultar muy cuestionables, lo interesante es observar cómo los niños tuvieron un papel fundamental para la comprensión antropológica de la especie humana y su desarrollo cultural. A diferencia de la tradición norteamericana, los antropólogos británicos vieron con desconfianza el acercamiento de la antropología a la psicología para comprender comportamientos no occidentales y optaron por centrarse en el estudio de las teorías funcional - estructuralistas, en las cuales los niños aparecían ocasionalmente en las discusiones sobre parentesco, crianza y ciclos de vida (LEVINE, 2007, pág. 251).

Una segunda línea importante de trabajo de la antropología británica fueron los estudios folkloristas y el interés de pensar a los niños como una "cultura tribal”. En las últimas décadas del siglo XIX y hasta 1940 la relación entre antropología y los estudios sobre Folklore fue bastante estrecha y aunque este tipo de estudios fue perdiendo popularidad, uno de los trabajos que directamente preguntó por la vida cotidiana y material de los niños fue la investigación de los esposos Iona y Peter Opie. En su trabajo de campo (1946 - 1969) en jardines infantiles y escuelas primarias de Inglaterra, Gales y Escocia, los Opie analizaron los juegos, las canciones, las rimas y las historias de los niños. Para estos antropólogos estas eran manifestaciones de una "cultura tribal infantil" separada del mundo adulto y que podría dar pistas sobre la manera en que la cultura se transmitía de un niño a otro.

Los Opie hablaron de la "sabiduría de los niños" en relación a sus propios mundos y por ello, los consideraron los principales informantes sobre las experiencias culturales y materiales del mundo infantil. Aunque este trabajo es considerado un antecedente teórico clave para la antropología de la infancia, también ha sido criticado por su enfoque. Por un lado, los Opie en su interés por resaltar y darle validez a las prácticas de los niños y sus juegos, separaron el mundo adulto del mundo infantil y en esta medida dejaron de lado las interacciones de los niños con el mundo social. En segundo lugar, si bien las observaciones y el ejercicio de recopilación de la información sobre la cultura material y folclórica de los niños fue un trabajo novedoso, los Opie no consultaron a los niños sobre por qué lo hacían o cómo lo experimentaban: "aprendemos poco sobre lo 
que esta "sabiduría" podría significar para los propios niños, o qué podría estar en juego para ellos en su uso" (ALLERTON, 2016, pág. 4).

La tradición norteamericana tomó una dirección diferente. Franz Boas rechazó cualquier comparación de los niños con los estados salvajes y primitivos de los grupos humanos, también cuestionó las ideas de una escala evolucionista en el que los niños y las comunidades primitivas representaban el nivel inferior de la evolución humana, mientras las sociedades occidentales europeas se presentaban como el ideal civilizatorio. Boas inició la trayectoria de investigación etnográfica sobre la infancia en la antropología estadounidense a través de sus estudios antropométricos sobre el crecimiento infantil entre los inmigrantes europeos en los Estados Unidos (LEVINE, 2007, pág. 249).

Como representante de la Escuela del Particularismo Histórico, educó a varios antropólogos que se convertirían, a partir de la segunda década del siglo $\mathrm{XX}$, en la primera generación de la Escuela Cultura y Personalidad: Ruth Benedict, Margaret Mead y Edward Sapir. Estos, al igual que su maestro, rechazaron cualquier reducción del niño al estado primitivo y se enfocaron en tratar de comprender la diversidad cultural de los modos de crianza y cuáles eran los efectos en la personalidad y el comportamiento adulto. En una de sus críticas a la asociación del niño con los primitivos, Margaret Mead señaló: "a pesar de la enorme cantidad de investigaciones que han demostrado la gran complejidad y diversidad de las culturas pre-alfabetizadas y la imposibilidad de cualquier concepto como "el primitivo", este todavía se repite con frecuencia monótona en la escritura psicológica” (MEAD, 1931, pág. 669).

No fue gratuito que parte de las investigaciones de Boas, Mead, y Benedict se hayan centrado en temas como la niñez y la adolescencia. La elección de estos temas fue un camino teórico para cuestionar el evolu- cionismo y para mostrar cómo la cultura moldeaba a sus individuos, desde el momento de su nacimiento, es decir, "cómo los niños devenían en seres culturales y cuál era el impacto que las primeras experiencias de la infancia tenían tanto en la personalidad adulta, como en el conjunto de la cultura de la sociedad a la que pertenecían" (PACHÓN, 2009, pág. 442).

Sin embargo, pese a la cercanía con la psicología, Benedict y Mead, al igual que en su momento lo hizo Malinowski, cuestionaron algunas de las posturas propuestas por la psicología del desarrollo y el psicoanálisis, especialmente las generalizaciones en contextos no occidentales de teorías como el complejo de Edipo, las etapas de desarrollo cognitivo de Piaget, las fases de desarrollo psicosexual de Freud, así como los roles sociales que se les asignan a los niños en las sociedades occidentales. Sobre este último punto, por ejemplo, Benedict a través de varias observaciones etnográficas, planteó que era necesario reevaluar las dicotomías, jerarquías y roles entre adultos y niños, cuestiones como: los adultos son dominantes y los niños sumisos y obedientes, los niños deben jugar y los adultos trabajar. En uno de sus trabajos más importantes sobre el tema afirmó: "todos estos son dogmas de nuestra cultura, dogmas por fuera de los hechos naturales, que otras culturas no comparten” (BENEDICT, 1938, págs. 21 - 22).

Por una línea similar de análisis, Margaret Mead, alumna de Benedict, escribió varios trabajos en los que se interesó por entender cómo se moldea el carácter de los niños y jóvenes "por obra de los diversos marcos culturales dentro de los cuales nacen los seres humanos, maleables y desprovistos de cultura" (MEAD, 1928a, pág. 13). Entre las investigaciones más importantes están "Adolescencia y cultura en Samoa" (1928a), "Samoan children at work and play" (1928b) "Childhood in Contemporary Cul- 
tures" (1954) y “Cultura y Compromiso" (1970). En estos trabajos ricos en descripciones etnográficas y uso de metodologías diversas (observación participante, entrevistas, tests, encuestas, dibujos y uso de instrumentos como cámaras y filmadoras), Mead buscó revaluar muchos de los supuestos "universales" sobre los patrones de comportamiento de los niños y adolescentes.

En sus primeros trabajos en Samoa (1928a), (1928b), argumentó que la educación de los niños configuraba la personalidad de los adultos y por tanto, la experiencia de la adolescencia variaba entre las culturas. A diferencia de la "turbulenta" y "conflictiva" adolescencia norteamericana, en Samoa el tránsito de la niñez a la adultez era una etapa tranquila. La adolescencia como quiebre no existía en Samoa, como en otras sociedades (MEAD, 1928a). En relación a los niños en Samoa (MEAD, 1928b), esta autora también hizo un trabajo de comparación entre las actividades de los niños y las relaciones con sus padres. Afirmó que en Samoa ningún padre consideraba que los niños no debieran trabajar, más bien el trabajo se convertía en un tipo de juego, pues las niñas cuidaban bebés de verdad y los niños aprendían tempranamente a construir casas reales. También, contrastó sus observaciones con las prácticas de crianza de la sociedad norteamericana de su época: "los padres de Samoa no esconden nada a sus niños. Ellos no les cuentan cuentos de hadas sobre el nacimiento de los bebes, ni tampoco los alejan cuando hay un funeral (...) Y los niños crecen sabiendo los ritmos de la vida y de la muerte, aceptando la vida como simple y sin rebeldía como lo hacen sus padres" (MEAD, 1928b, pág. 626).
En trabajos posteriores, Mead se preguntó por las relaciones de aprendizaje entre las generaciones y formas de transmisión cultural a las que denominó culturas posfigurativa, cofigurativa $\mathrm{y}$ prefigurativa (MEAD, 1970) y en 1954 formuló uno de sus proyectos más ambiciosos con otros antropólogos y psicólogos como Martha Wolfenstein para estudiar las diferentes formas de crianza infantil en diferentes sociedades: balí, judía, rusa, norteamericana, alemana y francesa (MEAD, 1954). En este trabajo, a diferencia de sus investigaciones previas, Mead y sus colaboradores, integraron modestamente las opiniones de algunos niños en sus análisis, también registraron los dibujos y pinturas y usaron algunas interpretaciones de los niños para su argumentación. Aunque la mayoría del libro privilegió la observación distante y los testimonios de adultos (padres, madres, maestros y expertos) se podrían considerar una novedad metodológica para el momento, cuando la mayoría de trabajos y descripciones etnográficas muy poco reconocían las voces de los niños como fuentes etnográficas autorizadas (MEAD, 1955). De ahí, que con excepción del trabajo de Mead y Wolfenstein, el interés de estos trabajos etnográficos no haya sido pensar a los niños como los principales informantes, sino a los adultos que los ayudarían en su proceso de socialización.

Así, durante las primeras décadas del siglo XX la escuela norteamericana Cultura y Personalidad y, en general, la antropología norteamericana tuvo una mayor influencia y trayectoria en el estudio de la infancia, que la escuela británica. Esto también le supuso un conjunto de críticas como el énfasis de estos trabajos en los procesos de socialización, así como el uso de categorías como "patrones culturales", "personalidad social"

${ }^{2}$ Este término hace referencia a la configuración de los rasgos o unidades funcionales y de los complejos culturales que constituyen una cultura determinada, en un momento dado.

${ }^{3}$ Se puede comprender esta noción en el sentido de que habían variaciones del temperamento humano configuradas de manera particular por cada sociedad, por lo que no se puede hablar de temperamentos innatos de cada sexo determinados biológica o fisiológicamente. 
"configuracionismo"4 para mostrar que había que distinguir modelos de temperamentos en la especie humana, de los que cada cultura hacía su propia selección para configurar sus modelos ideales. Muchos antropólogos cuestionaron el uso de estas nociones, cuando lo que se quería mostrar era la diversidad cultural, más que la definición de esquemas culturales cerrados. En su momento, Mead y Benedict respondieron a estas críticas afirmando que los individuos estaban en capacidad de tomar decisiones, salirse de los esquemas de personalidad ideal, pues no debían considerarse autómatas de los decretos de la civilización, ni la evolución (MEAD, 1939, pág. 31).

Aun cuando cada escuela de pensamiento antropológico realizó diferenciadamente sus primeros acercamientos al estudio sobre los niños, la contribución de la disciplina, en su conjunto, ha sido fundamental para la construcción de un campo de estudios sociales sobre infancia. En respuesta a los postulados filosóficos universales sobre la infancia, así como a las teorías biológicas y psicológicas sobre el desarrollo, el crecimiento, la socialización y la enculturación de los niños, varios antropólogos pioneros a través de su evidencia etnográfica mostraron la relatividad y variación cultural de las formas de crianza, las relaciones entre niños y adultos, las formas de aprendizaje y los roles sociales de los niños en sociedades occidentales, como no occidentales.

\section{Un cambio de mirada: la antropología de la infancia}

La antropología de la infancia como enfoque de estudio se consolidó en las décadas de los setenta y ochenta del siglo XX y se comprendió como una nueva perspectiva que "se soportaba en la noción de que la visión de los niños y su comprensión debería ser tomada seriamente y se rechazaba cualquier idea de que los niños fueran de algún modo incompletos e incompetentes" (MONTGOMERY, 2009, pág. 44). Muchos son los temas que actualmente se exploran a través del trabajo etnográfico y que han superado los marcos temáticos anteriores de la familia, la crianza, el parentesco y la vida primitiva. Actualmente, figuran en la agenda investigativa antropológica asuntos relacionados con prácticas escolares, consumo, trabajo infantil, enfermedad infantil, niños en la guerra, entre otros.

Después de la segunda mitad del siglo XX, los estudios antropológicos interesados en la vida de los niños tuvieron varios cambios tanto metodológicos, como teóricos, influenciados por un conjunto de discursos políticos y académicos emergentes. Así como los primeros acercamientos antropológicos al estudio de la infancia se nutrieron fundamentalmente de algunos debates propuestos por la biología, la psicología y el psicoanálisis, esta segunda etapa de desarrollo académico se basó sobre todo en las nuevas teorizaciones propuestas por la disciplina histórica a través del trabajo inaugural de Phillipe Aries, los estudios feministas, el nuevo paradigma de la sociología de la infancia y, por supuesto, los debates políticos, producto de la Convención de los Derechos del Niño en 1989.

La consolidación de un campo antropológico centrado en la infancia tuvo varios antecedentes. Desde finales de la década del cincuenta, algunas antropólogas como la norteamericana Mary Ellen Goodman (1952, 1957, 1960) y la británica Charlotte Hardman (1973) 
llamaron la atención sobre la importancia de tomar a los niños como "informantes antropológicos" (GOODMAN, 1957, pág. 979) y superar el confinamiento del niño a las estructuras familiares de socialización, desarrollo y parentesco. Goodman hizo un trabajo comparativo entre los valores de los niños norteamericanos y japoneses con respecto a las aspiraciones ocupaciones y profesionales. En su análisis tuvo en cuenta no solo la edad, sino las diferencias de género y su contexto nacional y, adicionalmente, precisó la importancia metodológica de registrar en la investigación las opiniones de los niños y niñas en la investigación: "ellos pueden decirnos de primera mano y sin retrospectiva lo que es su cultura a través de sus ojos, o lo que la niñez es a través de sus percepciones sobre la sociedad y la cultura. El concepto de niño como informante no es nuevo, aunque rara vez se ha explicitado y se ha utilizado mínimamente" (GOODMAN, 1957, pág. 979).

Esta antropóloga propuso varias estrategias metodológicas para trabajar con niños y así, aprender de los informantes infantiles como entrevistas, juegos, talleres con imágenes y construcción de relatos. Sostuvo que lo que los niños dicen no debía ser comprendido como un razonamiento periférico o complementario, tampoco debían considerarse como parte del "paquete de la dinámica psicológica o del proceso de la socialización", sino como "miembros fundamentales y portadores de la cultura" (GOODMAN, 1960, pág. 137), tan autorizados y calificados como sus mayores para hablar de sus sociedades.

Por esta misma línea, una década después, la antropóloga británica Charlotte Hardman aunque reconoció los trabajos pioneros de los primeros antropólogos británicos y norteamericanos como esfuerzos importantes para acercarse a la infancia, sostuvo que estos primeros acercamientos vieron a los niños “en mayor o menor grado como objetos pasivos, como espectadores en un ambiente con efectos en sus comportamientos. Ellos vieron a los niños como sujetos continuamente asimilados, que aprendían y respondían a los adultos, teniendo poca autonomía y contribuyendo poco a los valores sociales y a los comportamientos. Mi aproximación es estudiar a los niños como personas en su propio derecho y no como receptáculos de las enseñanzas adultas" (HARDMAN, 1973, pág. 504).

Pero, ¿cuáles fueron los discursos políticos y académicos que hicieron posible la emergencia de estas nuevas posturas antropológicas sobre los niños como las presentadas por Goodman y Hardman? En primer lugar, las reflexiones sobre infancia que se estaban configurando después de la segunda mitad del siglo XX no solo en la antropología, sino en las Ciencias Sociales, estuvieron influenciadas por la obra "Centuries of Childhood" (1962) del historiador francés Philippe Aries. Pese a las críticas que han surgido sobre esta obra (DEMAUSE, 1974), (POLLOCK, 1990) actualmente es innegable el peso de las tesis de este historiador para la consolidación del campo de estudios sociales sobre infancia y para la antropología de la infancia. Aries planteó desde una perspectiva histórica, que la noción de "infancia" no se podría considerar un descriptor de una fase biológica de la especie humana, sino que debía ser comprendida como una categoría histórica y políticamente contingente.

Para Aries, los sentimientos hacia la infancia no eran una cuestión "natural” a la especie humana, sino una manera en que las diferentes sociedades interpretan, comprenden e institucionalizan su compromiso con los niños, por lo que la infancia varía considerablemente entre culturas. Al analizar el caso europeo, especialmente a través de las pinturas, Aries afirmó que "el arte medieval hasta el siglo XII no conocía la infancia o no intentaba retratarla. Es difícil creer que este abandono 
se debió a la incompetencia o la incapacidad. Parece más probable que no haya lugar para la infancia en el mundo medieval” (ARIES, 1962, pág. 31). Según Aries, solo hasta el siglo XVII en el contexto europeo valió la pena hablar, escribir y pintar sobre los niños, periodo en el cual los niños se convirtieron en una nueva fuente de placer, de alegría y afecto para los adultos.

En este sentido, Aries reforzó desde la disciplina histórica varios de los argumentos que desde el trabajo de campo etnográfico, los primeros antropólogos de la Escuela Cultura y Personalidad, habían señalado en sus investigaciones con respecto a la variación cultural de la infancia y cómo la inversión emocional parental en los niños no era un hecho biológico incuestionable, sino que "cada cultura desarrolla su propia visión de la naturaleza de los niños, y con ella unas creencias sobre la mejor manera de cultivar esta "naturaleza" (SCHWARTZMAN, 1979, pág. 9). En segundo lugar, este historiador propuso que el estudio de las experiencias de los niños no podía desligarse de las experiencias del mundo adulto, dependen la una de la otra, de ahí que pensar a los niños como "tribus culturales" separadas de la vida social, no funciona para el análisis.

En suma, el trabajo de Aries reconoció que aunque la infancia es una etapa de desarrollo del curso de la vida común a todos los niños y caracterizada por cambios físicos, al mismo tiempo, es una noción histórica fragmentada por la diversidad de la vida cotidiana de los niños y depende de "las necesidades y competencias específicas de los niños que se ponen en manifiesto en la ley, la política social, así como en las interacciones sociales más cotidianas que tienen lugar entre adultos y niños" (James; James, 2001, pág. 27). De esta manera, Phillipe Aries abrió una agenda de investigación para el emergente campo de estudios sociales sobre infancia que se consolidaría en las décadas del setenta y ochenta. Algunos de los temas que más comenzaron a trabajarse fueron: los cambios a través del tiempo de la noción de infancia; la transformación de las sensibilidades y el trato de los adultos hacia los niños; la relación de la infancia con la institución escolar y la importancia de seguir el rastro histórico de los niños a través de la cultura material.

Otra importante influencia académica que tuvo el emergente enfoque de la antropología de la infancia fueron algunas ideas provenientes de los estudios feministas. Hardman, por ejemplo, retomó la noción feminista de "voces silenciadas" (ARDENER, 1975) para llamar la atención sobre los modos en que las mujeres y los niños habían sido construidos y entendidos de manera similar en la antropología y cómo el estudio de los mundos femeninos y las relaciones de género podían ayudar a comprender las experiencias de los niños. Para Hardman, las discusiones que se dieron desde la antropología feminista durante las décadas de los sesenta y setenta en las que se argumentaba la necesidad de incluir a las mujeres y a sus voces en las descripciones etnográficas y en la teoría antropológica, se estaban produciendo de manera similar en los setenta y ochenta con respecto a los niños.

Igualmente, se argumentaba que en las teorías sociales que dominaron mucho tiempo la discusión disciplinaria como la socialización, el parentesco y la psicología del desarrollo, los niños, como las mujeres habían sido históricamente pensados como adultos incompletos o incompetentes o como parte de las relaciones y dinámicas familiares (KEHILY, 2005, pág. 466), más que ser pensados como sujetos en sus propios términos y poseedores de diferentes competencias. Además, se puso de relieve la dimensión de género que atraviesa la infancia, para comprender que la diversidad cultural también se debía entender en las maneras en que ser niño o niña cambia la experiencia infantil: "la historia 
de la niñez y de la infancia se desdobla en la historia de cada género. Innumerables avatares por los que transita la niña son propios de su género y hablan de otras historias de la historia" (COLANGELO, 2003, pág. 4).

Aunque tal como sugirió Hardman, las discusiones antropológicas sobre el feminismo y sobre la infancia tuvieron varios puntos de encuentro, también en algunos aspectos se produjeron desavenencias. La antropóloga Jane Helleiner (1999) ha mostrado cómo el discurso sobre los niños se ha transformado en el pensamiento feminista. En la primera ola, el movimiento de mujeres norteamericano, de finales del siglo XIX y principios del siglo $\mathrm{XX}$, se interesó en la infancia, pero en el contexto de los roles maternales femeninos. A finales de los años sesenta, el discurso feminista sobre la infancia tomó otros matices, pues las categorías de "raza" y "clase" se convirtieron en variables de análisis fundamentales sobre las diferencias en la maternidad de grupos de mujeres de clases bajas, medias y altas de diferentes grupos étnicos. Así, mientras algunas activistas tenían opiniones positivas sobre la maternidad, otras la consideraban una práctica opresiva contra la mujer y por ende, se pensaba a los niños como sujetos que limitaban a las mujeres. De esta manera, "las discusiones feministas de la madre han tendido a reproducir las suposiciones culturales predominio-occidentales sobre la pasividad y la dependencia de los niños frente a las madres omnipotentes” (HELLEINER, 1999, pág. 28).

A diferencia de estos primeros enfoques, la antropología feminista posterior a la década del ochenta ha propuesto tener un compromiso más amplio con el estudio de la infancia por fuera de los marcos de la maternidad y pensar que las reflexiones sobre el género pueden ser un modelo analítico clave para pensar las experiencias infantiles y las relaciones generacionales entre adultos y niños. De igual forma la antropología de la infancia ha propuesto repensar la edad como una categoría fundamental también para los estudios feministas, pues "si bien el género es para la mayoría de los individuos una identidad y la infancia es por definición un estado temporal y de transición, reconocer que es temporal no significa devaluarlo" (WOODHEAD, 2009, pág. 29).

Además de los estudios de género, una de las fuentes más importantes de las que se ha nutrido la antropología de la infancia fue el nuevo paradigma de la sociología de la infancia que nació paralelamente a finales de los años ochenta en el contexto británico, con exponentes como Allison James, Chris Jenks, Alan Proust, Berry Mayall, William Corsaro, Adrian James y Leena Alaen. A pesar de que algunos antropólogos como Mead, Goodman y Hardman ya habían planteado, algunas décadas atrás, la importancia de pensar a los niños como informantes y participantes claves en el trabajo etnográfico, el nuevo impulso que dio la sociología de la infancia fue la conceptualización política de la infancia, que coincidió con el contexto de los debates sobre la Convención de los Derechos de los Niños (1989).

Estos sociólogos comenzaron a cuestionar las antiguas fórmulas teóricas sobre el estudio de la infancia, especialmente los paradigmas predominantes de la socialización y la psicología del desarrollo. Para estos investigadores era necesario superar estos paradigmas que moldeaban a los niños como objetos pasivos y víctimas de las influencias externas a ellos, incapaces de resistir y excluidos de participar en su propia socialización y a la infancia como una forma universal y determinada por la biología. Las propuestas de estos investigadores estuvieron enfocadas en pensar la infancia como un fenómeno social que se experimenta de manera diversa de acuerdo al contexto histórico, al lugar de origen, al género, la raza y la etnia (JAMES, 2003, pág. 28). También, se invitó a los científicos sociales a ex- 
plorar las perspectivas infantiles, reconocer las voces de los niños y pensar en términos de agencia y participación infantil. La sociología codificó estos planteamientos como "un nuevo paradigma social" (JAMES et al, 1998, pág. 6) y se señalaron algunos puntos de partida teóricos para la nueva investigación centrada en la infancia no solo desde la sociología, sino como propuesta interdisciplinaria: "la primera premisa es que la infancia se construye socialmente, la segunda es que los niños merecen ser estudiados por derecho propio y la tercera es que los niños son actores sociales competentes que pueden tener una perspectiva particular en el mundo social que nosotros, como adultos, puede ser que valga la pena escuchar” (James; James, 2001, pág. 26).

Esta discusión académica de la sociología de la infancia se materializó también en la agenda política internacional. Si bien desde la segunda década del siglo XX, después de la Segunda Guerra Mundial, varios países firmaron previamente dos tratados internacionales (Declaración de Ginebra de los Derechos del Niño1924 y Declaración de los Derechos del Niño -1959), solo hasta 1989 la Convención de los Derechos del Niño se estableció como un tratado internacional con carácter vinculante, en el que firmaron 192 estados reconocidos por la Asamblea Nacional de las Naciones Unidas con excepción de Estados Unidos y Somalia.

La Convención, en su forma básica, incluyó tres principios entrelazados: protección, provisión y participación. Los dos primeros han sido objeto del esfuerzo fundamental de organismos internacionales y grupos de derechos humanos, sin embargo, el principio de participación reforzó las ideas sobre agencia infantil que venían promoviéndose con el paradigma de la sociología de la infancia y también con algunos trabajos antropológicos como el de Hardman y Goodman. Además, este apartado tuvo efectos importantes en las reflexiones sobre la forma de estudiar la infancia, como el hecho de “evitar la objetivación de los niños dentro de las tradiciones de la investigación científica y fomentar la investigación con los niños, incluida la búsqueda de maneras en que los niños contribuyen al proceso de investigación como participantes, en algo más que un sentido simbólico" (WOODHEAD, 2009, pág. 25).

Aunque la Convención significó, en términos de política pública, un esfuerzo valioso para mejorar las condiciones de vida de muchos niños y niñas en el mundo y también fue un enorme paso en el reconocimiento de los niños como sujetos de derecho y de participación política, también se ha reconocido que desde su publicación ha generado dilemas difíciles en relación a su interpretación y aplicación en contextos no occidentales. La antropología ha sido, probablemente, la disciplina social más crítica con referencia a las implicaciones que tiene la imagen de infancia promovida por la Convención y que contradice, en gran parte, lo que por décadas la investigación etnográfica sobre la infancia ha intentado mostrar: la enorme diversidad y complejidad cultural de los mundos infantiles. A continuación, se señalarán algunas de las tensiones más importantes entre la Convención y la perspectiva antropológica contemporánea.

En primer lugar, la Convención está basada en el marcador de la edad para definir la infancia. Según el documento, se establece que un niño es toda persona menor de 18 años, sin embargo, desde la antropología se ha mostrado que la infancia no depende de una categoría como la edad, sino del significado social que internamente cada sociedad le adjudique a esta etapa de la vida. Para la antropóloga Heather Montgomery, “establecer en una edad específica el límite entre la infancia y la edad adulta no tiene mucho sentido para millones de niños que no tienen registro de nacimiento, ni manera de conocer sus edades. Para ellos, la cuestión de si son niños o no debe ser siempre una cuestión de 
conjeturas, habiendo otros marcadores como el matrimonio o el nacimiento de un hijo como marcadores de la edad adulta" (MONTGOMERY, 2009, pág. 64). Adicionalmente, no hay un consenso legal sobre en qué momento se empieza a hablar de niño, pues algunos países consideran que la infancia inicia en la concepción, otros en el nacimiento y en otras culturas como en la Isla de Bali, en Indonesia, consideran que el niño es humano 210 días después de nacido.

Un segundo punto es que la antropología ha cuestionado la idea de que todos los niños, donde quiera que vivan, tienen igualdad de derechos. La idea que se privilegia en la Convención sobre los niños como seres que esencialmente deben estudiar y no trabajar, deben ser protegidos en el contexto de una familia y tienen derecho a recrearse y a consumir, ha causado bastantes problemas para su interpretación e implementación en contextos no occidentales, ni urbanos. Para muchos antropólogos interesados en la infancia, los niños y niñas con los que se encuentran cotidianamente en su trabajo etnográfico no cumplen alguna o ninguna de estas características. Muchos son niños trabajadores, soldados, trabajadores sexuales, cuidadores, refugiados, desplazados, niñas madres y se casan tempranamente.

Así, antropólogas como Nancy Scheper, Myra Bluebond Langer, Heather Montgomery, Olga Nieuwenhuys, entre otros, han contribuido con sus investigaciones etnográficas al debate sobre los efectos de las políticas públicas en la vida de los niños y han argumentado la necesidad de que la legislación internacional sea flexible y se ajuste a los contextos locales y a las experiencias de vida de muchos otros niños que salen de estos contextos. Por ejemplo, la antropóloga Peggy Froerer (2009) cita la etnografía de Inge Bolin (2006) quien estudió el caso de las prácticas crianza de los niños de los Andes peruanos y señala que la inves- tigadora muestra que "en esta cultura la noción de infancia no existe como fase autónoma. Los niños de Chillihuani deben participar en tareas adultas y adquirir conocimientos de adultos sobre el parto, la medicina, la ganadería y la agricultura desde una edad muy temprana; no tienen tiempo para aprender las historias, las canciones y las poesías infantiles específicas de los niños, ni se dedican exclusivamente al "mundo del niño" (FROERER, 2009, pág.14).

Por último, una de las tensiones más evidentes que tiene la antropología está relacionada con la idea de "participación”, “competencia” y “agencia” que es promovida tanto por la Convención, como por el paradigma de la sociología de la infancia. Así como los estudios antropológicos han presentado interrogantes sobre enfoques como la psicología del desarrollo y la socialización, que asumen una progresión universal desde la infancia hasta la edad adulta, también cuestiona la idea universal de agencia, competencia y participación infantil, tal como se plantea en la Convención. Si bien los antropólogos, durante décadas, han documentado las formas de participación, acción y decisión de los niños en diferentes circunstancias, "lo que es menos claro es el grado de agencia, el impacto de esa agencia y mucho menos la naturaleza de esa agencia. Los niños como los adultos, no escapan a las limitaciones estructurales" (MONTGOMERY, 2009, p. 242).

En este sentido, la antropología también ha mostrado que no todas las sociedades comprenden a los niños como sujetos de derecho o como miembros competentes. Por ejemplo, Montgomery (2009) que ha descrito el trabajo del investigador Twum - Danso ha señalado cómo en el Japón la introducción de los “derechos de los niños" no fue una idea muy bien recibida, pues implicaba emplear nuevas nociones como individuo e individualismo, que en este contexto, ha tenido conexiones fuertemente negativas y se asocia con el egoís- 
mo: "los padres creían que los derechos de los niños conducirían a la división dentro de las familias y harían que los niños se volvieran egoístas y descuidaran a sus padres y a sus responsabilidades"(MONTGOMERY, 2009, pág. 69). También, en sus propios estudios etnográficos con niñas y adolescentes prostitutas en Tailandia, esta antropóloga ha reflexionado sobre la dificultad de hablar en términos de agencia, en el sentido como es comprendido por la Convención, cuando en este caso la fuente primaria de ingreso familiar proviene del trabajo de las niñas y ellas dan razones para hacerlo, no son obligadas a prostituirse, y afirman que "se quieren convertir en prostitutas por el deber y el amor a sus padres, que tienen una deuda moral, un deber conocido en tailandés como bunkhum" (MONTGOMERY, 2001, pág. 82).

También, antropólogos como David Lancy y Robert LeVine han mostrado varias dificultades de pensar en la noción de agencia infantil en la investigación social. Por un lado, LeVine sostiene que fácilmente la cuestión de la agencia infantil se puede convertir en parte de la agenda política y de defensa ideológica para el tratamiento de los niños y perder la perspectiva de la investigación académica que busque el entendimiento de ciertas realidades sociales (LEVINE, 2004, pág. 151). David Lancy, por su lado, muestra que la cuestión de la agencia infantil es un fenómeno digno de toda atención y estudio social, sin embargo, puede ser una noción etnocéntrica, clasista y hegemónica que puede negar la diversidad y la complejidad cultural que la antropología intenta mostrar (LANCY, 2012, pág. 4).

De esta manera, la Convención de 1989 se ha convertido en un poderoso instrumento para las políticas públicas y en un símbolo del compromiso de los estados modernos con la infancia y con los valores humanistas, liberales y democráticos. También, ha sido el escenario de promoción y apertura de la investigación académica sobre la infancia y con ella, la promesa intelectual y política de posicionar a los niños como actores sociales. Sin embargo, como lo ha sugerido Alisson James, la retórica de la participación, de la igualdad de derechos y de "dar voz a los niños", "no es simplemente permitir que los niños hablen" (JAMES, 2007, pág. 267), sino que tiene, en el fondo, unos desafíos metodológicos y epistemológicos que entran constantemente en tensión con los múltiples registros etnográficos que, desde finales del siglo XIX y durante todo el siglo XX, los antropólogos han presentado sobre las diferentes experiencias infantiles. La tarea desde la antropología consiste en seguir desajustando las preconcepciones y universalismos sobre la infancia y sus presumidas características y roles sociales.

\section{Algunas perspectivas de la Antropología de la Infancia desde América Latina}

Aunque la mayoría de investigaciones de Antropología de la Infancia se han situado en contextos como Norteamérica, Inglaterra y algunos países nórdicos, después de la década del noventa del siglo XX, varios antropólogos Latinoamericanos se han interesado en comprender la vida cotidiana de los niños y sus familias en la región. También, como lo hicieron sus colegas en otras geografías, se han esforzado por preguntarse por cómo se ha pensado y concretado el hecho de ser niño o niña en contextos locales y han mostrado las particularidades culturales que han influido en los modos de crianza, aprendizaje y desarrollo de los niños. Así, a través de sus registros etnográficos han puesto en cuestión ciertas generalizaciones y planes de intervención de las políticas públicas internacionales y nacionales. En este sentido, la agenda de investigación se ha enfocado en reflexionar sobre ciertos temas de interés y que competen a las experiencias múltiples de infancia en el 
contexto latinoamericano, entre las que se podrían mencionar: el trabajo infantil, niños consumidores de drogas, niños que habitan en las calles, niños en el conflicto, relaciones escolares, etnoeducación, formas de crianza y concepción de la infancia en las comunidades indígenas y otras minorías étnicas.

Por ejemplo, algunas antropólogas como la argentina Andrea Szulc y la brasilera Clarice Cohn han mostrado las diferentes maneras de comprender la infancia en comunidades indígenas latinoamericanas. Szulc en sus estudios sobre los niños mapuche de Argentina argumenta que la infancia es un campo de disputa entre diversos actores sociales (maestros, padres, Estado, legislación) que constantemente entran en tensión con respecto a qué es un niño, qué le corresponde hacer y qué no, qué actividades son las cotidianas, cómo se deben criar y cuidar y qué características se les adjudican. Mientras que las familias y las organizaciones mapuche han construido a los niños como sujetos competentes, responsables y por tanto, capaces de asumir responsabilidades relacionadas con la subsistencia, la producción y con la acción político - reivindicativa del grupo, los maestros creen que los niños mapuches "no tienen infancia" debido precisamente a su participación en este tipo de actividades. Por esto, para la comunidad la escuela más que "el lugar natural de la infancia", puede ser un espacio complejo y peligroso, pues allí se "desvaloriza a los niños" y les ofrecen un bajo nivel de instrucción práctico. En suma, existe una incomprensión recíproca respecto a cómo unos y otros comprenden la infancia (SZULC, 2011).

Por su lado, la antropóloga brasilera Clarice Cohn al estudiar el caso de los niños indígenas Xikrin en Brasil muestra que para la comunidad "un niño nada sabe porque todavía es niño, pero todo lo sabe porque todo lo ve y oye"(COHN, 2000, pág. 203). Aunque esto parezca contradictorio, la antropóloga encuentra que la comunidad considera que los niños no deben tener cierto tipo de responsabilidades peligrosas o penosas por su inmadurez física, tampoco pueden participar en actividades productivas y políticas, esto no se traduce en considerarlos miembros incompetentes de la sociedad. Por el contrario, desde que nace, el bebé puede participar activamente en la sociedad desde sus sentidos: su observación y su escucha les permite aprender de la vida social y ritual de la comunidad. Poco a poco, los niños tendrán más responsabilidades, como la cacería o las exploraciones, pero la infancia nunca es pensada como una etapa de incompetencia o carencia.

Otro de los grandes temas de discusión antropológica sobre la infancia es la contribución de los niños en las actividades productivas de su hogar y de sus comunidades. En Latinoamérica donde la mayoría de “contextos están marcados por las desigualdades sociales o la precariedad, los niños toman decisiones de manera individual o colectiva, participan en la elaboración de estrategias implementadas con los demás miembros de su familia y comunidad y asumen comportamientos adoptados a las circunstancias y a los problemas que los afectan" (CAVAGNOUD, et al. 2013, pág. 326). Así, antropólogos como Bogoña Leyra (2011) en México, Fernando Maureira (2007) en Chile, Patricia Ames (2013) en Perú y Zandra Pedraza (2007) en Colombia han estudiado las diferentes valoraciones sociales que pueden tener para las familias y los niños latinoamericanos la participación infantil en actividades productivas y domésticas.

La Convención de los Derechos del Niño (1989) proclamó que todos los niños del mundo debían retirarse de la esfera productiva e incluirse exclusivamente en los espacios de educación y recreo, sin embargo, para muchas familias latinoamericanas los escenarios de trabajo artesanal, agrícola, comercial, industrial y doméstico hacen parte fundamental de los procesos de ense- 
ñanza e integración de los niños a sus comunidades o en otros contextos, resulta fundamental para los presupuestos domésticos familiares y como estrategia de sobrevivencia. De ahí, que antropólogos como Pedraza, Leyra y Maureira llamen la atención sobre la importancia de analizar el trabajo infantil en el contexto latinoamericano a partir de la comprensión del sistema económico local y familiar del que el niño hace parte (MAUREIRA, 2007, pág. 279) y deconstruir los mitos sobre el trabajo infantil como un ejercicio necesariamente peligroso, producto de la pobreza y que hace a los niños vulnerables y los aleja de la esfera de la escuela (LEYRA, 2011).

Así, la Convención y otros organismos internacionales rechazan cualquier forma de trabajo infantil en estas regiones y se insta a reconocer al niño más como consumidor, que como productor, pero el problema se ha tratado de forma aislada "de las condiciones generales de trabajo de las familias de los niños y de las condiciones subordinadas de los grupos sociales a los cuales pertenecen (campesinos, trabajadores informales, entre otros) (...) Surge de esta reflexión la inquietud por la situación del niño como agente productivo: ¿es posible la niñez si los niños trabajan? ¿Son solo niños aquellos insertos como consumidores en la economía?” (PEDRAZA, 2007, pág. 86).

Por ejemplo, Patricia Ames estudia el caso de los Andes peruanos y señala que las transiciones de cambio y crecimiento de los niños no se comprenden desde marcadores occidentales como la edad o por algún rito de paso específico, "sino que muchas veces son procesos más sutiles, en los que se adquiere gradualmente los conocimientos y destrezas que permiten convertirse en un miembro adulto en el seno de la sociedad" (AMES, 2013, pág. 392). Ames hace referencia concretamente a los procesos de aprendizaje en la vida cotidiana, a lo que el niño es capaz o no de realizar en diferentes momentos de sus vida y a sus posibilidades de participar en cierto tipo de actividades productivas (agrícolas, domésticas, artesanales, crianza de hermanos). Estos "modelos autóctonos de desarrollo infantil" de las comunidades de los Andes peruanos, dice la investigadora, no deben pensarse como un conjunto de costumbres exóticas, "sino reconocerlos como parte de un conocimiento local coherente sobre el desarrollo infantil” (AMES, 2013, pág. 398).

Finalmente, un tercer tema de interés de la literatura antropológica sobre infancia en América Latina han sido las experiencias escolares y los procesos de aprendizaje de los niños. Algunas reflexiones se han enfocado en las formas de educación local de algunas comunidades indígenas (DÍAZ, 2010), (REMORINI, 2013), en las prácticas de educación corporal en las escuelas y la formación de ciudadanía (MILSTEIN y MENDES 2007) y las prácticas pedagógicas y las relaciones de los niños y sus familias con la escuela (CARLI, 1997). Entre estas investigaciones, mencionaré el análisis de la antropóloga colombiana Maritza Díaz en su estudio etnográfico sobre los procesos de enseñanza indígena con los Cubeo y Ticuna del Amazonas colombiano. Díaz muestra que por fuera de los marcos escolarizados occidentales, los niños indígenas de estas comunidades tienen unas formas de aprendizaje que pasan por la apropiación, uso y adaptación de prácticas sociales, materiales y herramientas simbólicas desarrolladas por sus culturas.

Así, al igual que Patricia Ames, Díaz reconoce que el crecimiento de los niños está más delimitado por acontecimientos y saber "hacer, observar y escuchar" con su cuerpo, que por ciclos de edad. Los niños aprenden a moverse, jugar, trepar y hacer diversos usos de su cuerpo a través de su relación con el medio. Los adultos no los alejan del peligro, ni los alertan sobre los riesgos, más bien formulan preguntas a los niños como ruta para 
entender y extraer los aprendizajes de las mismas acciones: “aparece aquí un principio muy importante en los procesos de enseñanza y aprendizaje. Se trata de permitir que el niño experimente las consecuencias de sus actos. Si bien el cuidado del adulto busca que el niño no salga lastimado, dentro de un margen de seguridad se deja que los niños establezcan la conexión de causa efecto para aprender" (DÍAZ, 2010, pág.71). Así, lo que muchos padres y maestros en contextos occidentales pueden reconocer como actos riesgosos y potencialmente dañinos para los niños, para las familias de estas comunidades indígenas es un espacio de aprendizaje por vía de la exploración directa. Algunos de los aprendizajes son anticipar efectos, valorar los saberes previos y ponerlos al servicio de sus acciones, validar los erro- res y aprender de ellos, tomar decisiones autónomas y generar criterios de autorregulación.

Las experiencias locales de la región también han nutrido las discusiones académicas mundiales sobre antropología de la infancia y han proporcionado información etnográfica valiosa sobre otras formas de infancia posible. Una deuda importante que se tiene en la región continúa siendo la importancia de involucrar a los niños más directamente en las investigaciones, como informantes y como investigadores. Son pocos aún los trabajos que presentan las perspectivas infantiles sobre sus mundos culturales y se sigue privilegiando las miradas de los adultos y de los mismos antropólogos en las investigaciones.

\section{Referencias Bibliográficas}

ALANEN, Leena. Rethinking Chilhood. En: Acta Sociológica, v. 3, no. 1, 1988, p. 53 - 67.

ALLERTON, Catherine. Introduction: Encontering Children. En: Children Ethnographic Encounters. London: Bloomsbury, 2016, p. 1 - 15.

AMES, Patricia. Niños y niñas andinos en el Perú: crecer en un mundo de relaciones y responsabilidades. En: Bulletin de l'Institut Français d'Études Andines, v. 42, no. 3, 2013, p. 389 - 409.

ATTARD, Sharon. Emerging Perspectives in the Anthropology of Childhood. En: Anthropology Today, v. 24 , no. 5, 2008, p. 24.

ARIES, Phillipe. Centuries of Childhood. New York: Alfred Knopf, 1962.

ARDENER, Edwin. Belief and the Problem of Women. En: Perceiving Women. London: J. M. Dent, 1975 , p. 1 17.

BENEDICT, Ruth (1934) Patterns of Culture. Boston, Massachusetts: Houghton Mifflin, 1989.

BENEDICT, Ruth (1938). Continuities and Discontinuities in Cultural Conditioning. En: MEAD, Margaret (ed) Childhood in Contemporary Cultures. Chicago: The University of Chicago Press, 1955, p 21 - 31.

BLUEBOND, Myra. Challenges and opportunities in the Anthropology of Childhoods: an introduction to Children, childhood and childhood studies. En American Anthropology, v. 109, no. 2, 2007, p 241 - 246.

BOAS, Franz (1911) La mente del hombre primitivo. Buenos Aires: Ediciones Solar, 1964.

CARLI, Sandra. Infancia, psicoanálisis y crisis de generaciones: una exploración de las nuevas formas del debate en la educación. En: PUIGRÓSS, Adriana (Dir), Dictaduras y utopías en la historia reciente de la educación argentina (1955 - 1983). Buenos Aires: Galerna, 1997. 
CAVAGNOUD, Robin; DE SUREMAIN, Charles-Edouard; GONZÁLEZ LA RIVA, Palmira. Introducción: Infancia y niños en las sociedades andinas contemporáneas. En: Boletín del Instituto Francés de Estudios Andinos, v. 42 , no. 3,2013 , p. $323-332$.

COHN, Clarice. Crescendo como um Xikrin: uma análise da infância e do desenvolvimento infantil entre os Kayapó-Xikrin do Bacajá. En: Revista de Antropología, v. 43, no. 2, 2000, p. 195 -222.

COLANGELO, María Adelaida. Ponencia: la mirada antropológica sobre la infancia. Reflexiones y perspectivas de abordaje. En: Laboratorio en Investigaciones en Antropología Social. Buenos Aires: La Universidad Nacional de la Plata, 2003, p 1 - 8.

CUNNINGHAM, Hugh. Histories of Childhood. En: The American Historical Review, v. 103, no. 4, 1998, p 1195 $-1208$.

DARWIN, Charles (1877). A biographical sketch of an infant. En: KESSEN, William (Ed) The Child. New York: Willey, 1965.

DEKKER, Jeroen. Philippe Aries's discovery of childhood after fifty years: the impact of a classic study on educational research. En: Oxford Review of Education, v. 28, no. 2, 2012, p.133 - 147.

DEMAUSE, Lloyd. La evolución de la infancia. New York: Phychohistory Press, 1974.

DÍAZ, Maritza. Contribuciones a la Antropología de la Infancia. La niñez como campo de agencia, autonomía y construcción cultural. Bogotá: Universidad Pontificia Javeriana, 2010.

FROERER, Peggy. Ethnographies of Childhood and Childrearing. En: Reviews in Anthropology, v. 38, no.1, $2009,3-27$.

GOODMAN, Mary Ellen. Children as informants: The Child's eye view of society and culture. En: The American Catholic Sociological Review, v. 21, no. 2, 1960, p. 1179 - 1187.

GOODMAN, Mary Ellen. Values, aptitudes and social concepts of Japanese and American Children. En: American Anthropologist, v. 59, no. 6, 1957, p. 979 - 999.

GREENE, Sheila, and HILL Malcolm. Researching Children's Experience: Methods and Approaches. Los Angeles: Sage, 2005.

HARDMAN, Charlotte. Can there be an anthropology of children? En: JASO v. 4, no. 2, 1973, p. 501 - 117.

HARDYMENT, Christina. Dream Babies: child care from Locke to Spock. Oxford: Oxford University, 1984.

HELLEINER, Jane. Toward a Femenist Anthropology of Childhood. En: Atlantis, v. 24, no. 1, 1999, p. $27-38$.

HIRSHFELD, Lawrence. Why don't anthropologist like children? En: American Anthropological Association Willey, v. 104, no. 2, 2002, p. $611-627$.

HOBBES, Thomas (1651) El Leviatán. Madrid: Alianza, 1999.

HOGAN, Diane. Researching the child in developmental psychology. En: GREENE, Sheila and HILL Malcolm. Researching Children's Experience: Methods and Approaches. Los Angeles: Sage, 2005, p. 22 - 42.

JAMES, Alisson. Giving voice to children's voice: practices and problems, pitfalls and potentials. En: American Anthropology, v. 109, 2007, p. 261 - 272.

JAMES, Alisson y JAMES Adrian. Childhood: toward a Theory of Continuity and Change. En: The Annals of the American Academy of Political and Social Science, v. 575, 2001, p 25 - 37.

JAMES, Allison. Understanding childhood from an interdisciplinary perspective. En: Peter B (ed) Rethinking Childhood. Brunswick: Rutgers University Press Language, 2003.

JAMES, Allison; JENKS, Chris y PROUST Alan. Theorizing Childhood. Cambridge: Polity Press, 1998.

JENKS, Chris. Constructing Childhood Sociologically. En: An introduction to childhood studies. New York: McGraw Hill, 2009.

KEHILY, Mary Jane. Gendered Childhoods. En: Gender and Education, v.17, no. 5, 2005, p. 463 -469. 
KEHILY, Jane. An introduction to childhood studies. New York: McGraw Hill, 2009.

KIDD, D. Savage Childhood: A Study of Kafir Children. London: Black, 1906.

LANCY, David. The Anthropology of Childhood: Cherubs, Chattel, Changelings. Cambridge: University Printing House, 2015.

LANCY, David. Why anthropology of childhood. A brief history of an emerging discipline. En: AnthropoChildren, v. 1, 2012, p. 1 - 17.

LANCY, David. Unmasking Children’s Agency. En: AnthropoChildren, v. 1, no. 2 2012, p. 1 - 20.

LEIRA, Begoña. Aproximaciones antropológicas a la infancia trabajadora: deconstruyendo los mitos y analizando los mitos de una compleja relación. En: JOCILES, M. I.; FRANZÉ, A.; Poveda, D. (Eds.). Etnografías de la infancia y de la adolescencia. Madrid: Catarata, 2011.

LEVI - STRAUSS, Claude. Antropología Estructural. New York: Basic Books, 1963.

LEVINE, Robert. Ethnography studies of childhood: a historical overview. En: American Anthropology, v. 109, no 2, 2007, p. $247-260$.

LOCKE, John (1690). Ensayo sobre el entendimiento humano. México: Fondo de Cultura Económica, 1999.

LOCKE, John (1693). La conducta del entendimiento. Madrid: Anthropos, 1992.

MALINOWSKI, Bronislaw (1929). Prenuptial Intercourse between the Sexes (The Sexual Life of Children, Age Divisions, the Amorous Life of Adolescence, and the Bachelor's House. En: The Sexual Life of Savages in Northwestern Melanesia. Honolulu: University Press of the Pacific, 2001.

MAUREIRA ESTRADA, Fernando. El trabajo infantil: una aproximación antropológica. En: Familia y Diversidad en América Latina. Estudios de casos. Buenos Aires: CLACSO, Consejo Latinoamericano de Ciencias Sociales, 2007, p. 275 - 285.

MEAD, Margaret (1928a). Prefacio a la edición de 1939. En: Coming of Age in Samoa. New York: Perennial Classics, 2001.

MEAD, Margaret. The primitive child. En: Handbook of Child Phychology, Worcester, Mass: Clark University Press, 1931.

MEAD, Margaret. Samoan Children at Work and Play. En: Natural History, v. 28, no. 6 1928b, p. 626- 636.

MEAD, Margaret (1970) Cultura y compromiso: el mensaje de la nueva generación. Barcelona: Granica Editor, 1977.

MEAD, Margaret. Childhood in Contemporary Cultures. Chicago: The University of Chicago Press, 1955.

MESSENGER, Davies. The study of Childhood. En: Children, Media and Culture. McGraw - Hill Education, 2010.

MILSTEIN, Diana y MENDES, Héctor. La escuela en el cuerpo: estudios sobre el orden escolar y la construcción social de los alumnos en las escuelas primarias. Buenos Aires: Niño y Dávila Editores, 2007.

MONTGOMERY, Heather. Gendered childhoods: a cross disciplinary overview. En: Gender and Education, v. 17, no. 5, 2005, p 471 - 482 .

MONTGOMERY, Heather. An introduction to childhood: anthropological perspectives on children's lives. Oxford: Wiley-Blackwell, 2009.

MONTGOMERY, Heather y WOODHEAD Martin. Understanding Childhood: and indisciplinary approach. Hoboken: Wiley and sons, 2003.

MONTGOMERY, Heather. Becoming part of this world: anthropology, infancy and childhood. En: JASO, v. 31, no. 1,2000, p 15 - 30 .

PACHÓN, Ximena. ¿Dónde están los niños: Rastreando la mirada antropológica sobre la infancia. En Maguaré, no. 23, 2009, 433 - 469. 


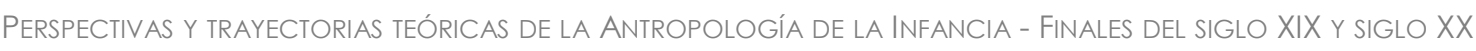

PEDRAZA, Zandra. El trabajo infantil en clave colonial: consideraciones histórico - antropológicas. En Revista Nómadas, no 26, 2007, p. 80 - 90.

PEDRAZA, Zandra. Al borde de la razón: sobre la anormalidad corporal de niños y mujeres. En: Cuerpos anómalos. Bogotá: Universidad Nacional de Colombia, 2008.

POLLOCK, Linda. Los niños olvidados: relaciones entre padres e hijos 1500 a 1900. México: Fondo de Cultura Económica, 1990.

REMORINI, Carolina. Estudios etnográficos sobre el desarrollo infantil en comunidades indígenas de América Latina: contribuciones, omisiones y desafíos. En: Revista Perspectiva, v. 31, no. 3, 2013, p. 811 - 840.

ROUSSEAU, Jacques (1762). Emilio o de la Educación. ERMIÑO, Mauro (ed). Madrid: Alianza Editorial, 1998.

SCHWARTZMAN, Helen. The invention of Childhood. En: Transformations: The Anthropology of Children's play. Chicago: Institute for Juvenile Research, 1979.

TYLOR, Edward (1871) Primitive Culture: Researches into the Development of Mythology, Philosophy, Religion, Language, Art and Custom. London: John Murray, 1913.

WALKERDINE, Valerie. Developmental pshychology and the study of childhood. En: KEHILY, Jane. An introduction to childhood studies. New York: McGraw Hill, 2009.

WHITING, B. Six Cultures: Studies of Child Rearing. New York: Wiley, 1963.

WOODHEAD, Martin. Child Development and the Development of Childhood. En: QVORTRUP, Jens y CORSARO, William. The Palgrave Handbook of Childhood Studies. United Kingdom: Palgrave, 2009.

SZULC, Andrea. Esas son cosas de chicos: disputas en torno a la niñez mapuche en el Neuquén, Argentina. En: JOCILES, M. I.; FRANZÉ, A.; POVEDA, D. (Eds.). Etnografías de la infancia y de la adolescencia. Madrid: Catarata, 2011.

Submissão: 27/04/2018

Aceite: $12 / 10 / 2018$ 